\title{
La gramaticalización de usted: un cambio lingüístico en proceso. Evidencias en el Uruguay del siglo XIX
}

\author{
Virginia Bertolotti ${ }^{1}$
}

RESUMEN: Estudio aquí la presencia/ausencia de usted sujeto y la alternancia del sintagma a usted con to o la objeto directo a lo largo del siglo XIX en Uruguay. El estudio del comportamiento de los miembros de la subclase de usted se contrasta con el de las otras posibilidades alocutivas singulares en el español en Uruguay (la subclase de tú y la subclase de vos). La interpretación de los datos se realiza a la luz del enfoque de la gramaticalización en relación con estudios de la cortesía y muestra las características del proceso de cambio el paradigma de usted durante el siglo XIX en Uruguay.

PALABRAS CLAVE: gramaticalización; usted; siglo XIX; Uruguay.

RESUMO: Estudo aqui a presença/ausência do usted sujeito e a alternância do sintagma a usted com lo ou la objeto direto durante o século XIX no Uruguai. O estudo do comportamento dos membros da subclase usted é contrastado com outras possibilidades alocutivas singulares no espanhol no Uruguai (a subclase de tú e a subclase de vos). A interpretação dos dados é feita a partir dos postulados teóricos da gramaticalização em relação com os estudos da cortesia. Ela mostra as características do processo de mudança do paradigma de usted durante o século XIX no Uruguai.

PALABRAS CHAVE: gramaticalização; usted; século XIX; Uruguai

1. Introducción

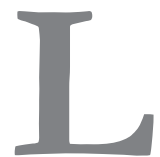

a clase de los pronombres personales de segunda persona singular en español está constituida por los nominativos tú, vos, usted, los oblicuos ti, contigo, los acusativos te, lo, la y los

1 Universidad de la República, Agencia Nacional de Investigación e Innovación. virginia.bertolotti@gmail.com. 
dativos te, le $(s e)^{2}$. Esta clase ha sido objeto de gran cantidad de estudios por constituir un eje de variación del español, al combinarse de manera diversa dando lugar, a grandes rasgos, a cuatro sistemas pronominales con sendas distribuciones geográficas, tal como ha explicado, entre otros, Fontanella de Weinberg (1999).

Los pronombres de segunda persona o alocutivos son también analizados como formas de tratamiento, ya que codifican posiciones discursivas de los hablantes, y significados sociales, también sujetos a variación dialectal.

La comprensión cabal de la complejísima situación del sistema alocutivo del español actual, lengua hablada por más de cuatrocientos millones de personas, solo es posible a través de un análisis diacrónico conjunto de las subclases que constituyen el sistema. Sin embargo, en esta ocasión, me voy concentrar, sobre todo, en una de ellas: la de usted.

Estudiaré aquí la presencia/ausencia de usted sujeto y la alternancia del sintagma a usted con lo o la objeto directo a lo largo del siglo XIX en Uruguay $^{3}$. El estudio del comportamiento de los miembros de la subclase de usted será contrastado con el de las otras posibilidades alocutivas singulares en el español en Uruguay, las de la subclase de tú y las de la subclase de vos.

En primer lugar, sintetizaré el proceso histórico del surgimiento de la forma usted y las interpretaciones sobre este proceso (apartado 2). En segundo lugar, desarrollaré algunos conceptos teóricos necesarios para la comprensión del ordenamiento y análisis de los datos y describiré el corpus en el que me basé (apartado 3). Luego, presentaré e interpretaré los datos sobre la presencia de sujetos usted en contraste con los sujetos tú y vos, y los objetos directos a usted lo/la $\sim$ lo [verbo]a usted en contraste con los objetos directos expresados por te $\sim$ a ti/vos $\sim$ te Jverbo]a til vos (apartado 4). Interpretados los datos, justificaré por qué entiendo que la subclase usted se encontraba en proceso de cambio en el siglo XIX en el Uruguay y señalaré algunas implicancias teóricas y metodológicas (apartado 5).

2 Al usar el término "persona" estoy haciendo referencia a las personas del discurso locutor (primera persona), alocutario (segunda persona) y no participante (tercera persona), en el conocido sentido de Ducrot- y no a los rasgos de flexión verbal. 
2. La historia de usted y sus interpretaciones

El cambio que conocemos como el pasaje de la vuestra merced a usted, es, como todos los cambios lingüísticos, multicausal. Este cambio tiene aspectos fónicos, morfosintácticos, semánticos y pragmáticos, que han sido profusamente tratados en la bibliografía del español. En esta somera revisión, incluyo los tres trabajos principales, a mi juicio, en cuanto al origen y difusión del cambio en cuestión.

Koch (2008: 59-60) entiende que vuestra merced es ejemplo de uno de los procedimientos de "tratamiento nominal abstracto" que "se refiere directamente a una entidad abstracta más que al interlocutor mismo". Remonta este recurso a la antigüedad latina, como uno dentro de un conjunto de tradiciones discursivas ${ }^{4}$ ligadas a la carta. El recurso al nominal abstracto surge, según este autor, en el romance hispánico a través del contacto con la tradición diplomática propia de la cultura latina. Data las primeras apariciones de la vuestra merced en el siglo XIV en convivencia con otros sustantivos abstractos como señoría, majestad, alteza, excelencia.

En cuanto a la difusión de vuestra merced afirma Koch (2008: 70):

$\mathrm{Al}$ principio, lo que por supuesto no nos está atestiguado, vuestra merced es una opción dentro del procedimiento discursivo más general del tratamiento abstracto. Nace en cuanto tratamiento al rey, pero sigue el itinerario casi natural de todas las fórmulas de cortesía (igual que, por ejemplo, la pluralización del tratamiento y ciertos tratamientos abstractos de la Antigüedad tardía) y se extiende a la jerarquía eclesiástica, y, además, a posiciones sociales menos altas: condes, funcionarios, etc.

Este autor describe además, el proceso de idiomatización, es decir, el pasaje de las reglas discursivas a las reglas idiomáticas, identificando en dicho proceso una serie de factores (cf. Koch, 2008: 75 y ss.), de los cuales no es posible dar cuenta aquí en su totalidad. Retomo, sin embargo dos de ellos: la universalización: que entiende como el pasaje del dominio discursivo diplomático

3 Por razones de simplicidad en la expresión usaré el término Uruguay para referir al territorio al oriente del Río del mismo nombre, independientemente del período al que me esté refiriendo y de los diversos nombres que se ha le han dado en las diversas situaciones políticas regionales por las que ese territorio ha pasado.

$4 \quad$ "La noción de Tradiciones Discursivas [...] parte de la distinción coseriana de los tres niveles del análisis lingüístico [... y] pone énfasis sobre la tradición de los textos [...], se refiere a los textos ya producidos en una comunidad, el acervo cultural, la memoria textual o discursiva." (Kabatek 2008:9) 
al cortesano y la sistematización es decir, el desarrollo de concordancias de tercera persona.

La interpretación tradicional de Lapesa sobre el éxito de vuestra merced $>$ usted se relaciona con dos hechos. Por un lado, se asocia a un gusto ceremonial que había promovido el florecimiento de tratamientos abstractos en el siglo XV, muchos de los cuales se especializaron en interlocutores particulares como puede ser el caso de vuestra excelencia (duques, virreyes), vuestra señoría (obispos, condes y marqueses), vuestra alteza (príncipes) (Lapesa, 1970: 318). Por otro lado, se asocia al desgaste de vos, plural etimológico, que, usado para dirigirse a un solo interlocutor, constituía una forma de respeto o distancia (Lapesa, 1959:251, 1970: 149)5.

De Jonge y Niewenhuijsen (2009) realizan un detallado análisis de una abundante cantidad de textos literarios y gramáticas de época. Estos autores documentan las primeras apariciones de vuestra merced en el siglo XIV. En el siglo siguiente registran usos esporádicos, pero en el siglo XVI la forma vuestra merced o abreviaturas de ella aparecen ya abundantemente. A partir del análisis de las frecuencias así como de la interpretación de varios ejemplos postulan que la extensión ${ }^{6}$ del uso de vuestra merced se da a partir del siglo XVI, en lo que coinciden con los otros autores ya citados.

En cuanto al significante, De Jonge y Niewenhuijsen (2009: \15.7.3) documentan que en el siglo XVII el proceso de gramaticalización de sintagma nominal a pronombre está lo suficientemente avanzado: ya ha comenzado la pérdida del artículo la (se registra la vuestra merced pero también vuestra merced). En los siglos XVI y XVII avanzan sustancialmente la fusión de vuestra y merced y la reducción fónica que transformará vuestra merced en usted.

Tanto Koch como De Jonge y Niewenhuijsen apelan al concepto de gramaticalización para dar cuenta del proceso de cambio la vuestra merced $>$ usted. Existen diversas acepciones para el término gramaticalización que reflejan diversas posturas teóricas (para esa discusión sugiero Hopper \& Traugott, 1993: caps. 1 y 2, Heine, Claudi \& Hünnemeyer, 1991, capítulo 1). De acuerdo con Company (2003) se suele entender gramaticalización

5 En este mismo sentido, ver los trabajos de Cano Aguilar (1997: 244); Penny (1993:139); Kany (1945: 58-62).

6 Se da, en rigor, una generalización por extensión metafórica, con el sentido en que se usan estos dos términos en la bibliografía sobre gramaticalización 
en dos sentidos. El primero de ellos, planteado por Meillet (1912) y Kury ${ }^{3}$ owicz (1965:69), entre otros, es el siguiente:

un proceso mediante el cual una forma o construcción asume una función gramatical, o bien una entidad o construcción ya gramatical adquiere una función aún más gramatical” (Company, 2003:9)

Otra manera de entender gramaticalización es "como la creación o generación de gramática" (Hopper 1987, 1998). Para esta autora, Trauggot (1982, 1989, 2003) y Girón (2002a: 104-105) también lo entienden de esa manera. Este sentido, complementario del anterior, puede definirse así:

la fijación de estrategias discursivas, de manera que los fenómenos que, en un estado de lengua dado, operan en un nivel discursivo o textual, en un nivel más pragmático, se convierten con el paso del tiempo en construcciones gramaticales convencionales, carentes ya de condicionamientos pragmáticos (Company, 2003: 9)

El análisis de Koch integra la idea de que el pasaje de (la) vuestra merced a usted es una gramaticalización, sobre todo en este último sentido, apelando al concepto de tradiciones discursivas. El análisis de De Jonge y Niewenhuijsen (2009) apela al concepto de gramaticalización, aunque preferentemente en el primero de los sentidos. Sin mención explícita, la interpretación de Lapesa se inscribe también en el primero de los sentidos.

Sin embargo, ni ellos, ni otros autores hasta donde me consta, han insistido, como me interesa plantear aquí, que el proceso no concluye con el establecimiento de la forma fónica usted. Mostraré en el apartado 4 que el cambio continúa en proceso en el siglo XIX, por lo menos en Uruguay, ya que el comportamiento de la subclase de usted no es igual que el de las subclases de túy vos.

3. El corpus, las categorías conceptuales y el método de análisis

El corpus está constituido por 100 cartas personales seleccionadas de diversos archivos históricos ${ }^{7}$, que se dividen en dos sub-corpus con

7 Este corpus fue constituido con el fin de realizar mi tesis doctoral sobre formas de tratamiento en Uruguay en el siglo XIX, dirigida por Adolfo Elizaincín y Elvira Arnoux. Las cartas fueron elegidas entre las existentes en diversos repositorios: el Archivo General de la Nación de Montevideo en el cual se realizó la búsqueda en las Secciones 
iguales características y que solo se diferencian por el período al que pertenecen. Los períodos establecidos son los siguientes: siglo XIX 1 (17931830) y siglo XIX 2 (1872-1905). El hiato temporal entre ambas partes del corpus me permite evidenciar algunos aspectos del cambio lingüístico, al saltar por lo menos una generación.

Para asegurar la comparabilidad entre ambas partes del corpus tomé en cuenta nueve condiciones para su constitución. En esta ocasión, me limito a considerar dos de ellas, necesarias para la comprensión del análisis de los datos que presento en este artículo: el ámbito de relacionamiento de los bablantes y la relación de poder entre ellos.

Consideré dos tipos de ámbito de relacionamiento para los interlocutores de las cartas: familia, personas unidas por lazos de hasta segundo grado, y sociedad, personas unidas por una relación no familiar ${ }^{8}$.

En cuanto a las relaciones de poder, entiendo este término en el sentido en que es utilizado en el estudio clásico de Brown y Gilman (1960) ${ }^{9}$. Hago notar que no incluí el parámetro solidaridad ${ }^{10}$ postulado, no obstante conservo un aspecto de este concepto bajo la idea de igual poder. El tipo de datos requeridos para poder interpretar el parámetro solidaridad es difícilmente deducible a partir de textos epistolares muchas veces únicos, o no integrados a un epistolario, ya que estos se inscriben básicamente en la vida cotidiana y fueron escritos por personas cuyo detalle biográfico es casi por completo desconocido. Las relaciones de poder, por el contrario, sí son fácilmente deducibles del léxico y de las expresiones contenidas en las propias cartas.

Particulares, Judiciales y en el fondo del Ex Archivo Administrativo; el Archivo General de la Nación de Buenos Aires, el Archivo Saravia del Centro de Estudios Históricos del Comando General del Ejército; el Archivo de Indias; el Archivo Artigas y la Revista Histórica.

8 El desconocimiento biográfico de muchos de los autores de las cartas hace imposible establecer categorías más refinadas.

9 "One person may be said to have power over another in the degree that he is able to control the behaviour of the other. Power is a relationship between al least two persons, and it is nonreciprocal in the sense that both cannot have power in the same area of behaviour" Brown y Gilman (1960: 255).

10 La caracterización propuesta por Brown y Gilman (1960: 258) no es del todo esclarecedora: "Now we are concerned with a new set of relations which are symmetrical; for example, attended the same school or have the same parents or practice the same profession. (...) Solidarity is the name we give to the general relationship and solidarity is symmetrical. The corresponding norms of address are symmetrical or reciprocal with $\mathrm{V}$ becoming more probable as solidarity declines." 
Las bases del poder, según Brown y Gilman, se ligan a la fortaleza física, la riqueza, la edad, el sexo y los roles institucionalizados, como los establecidos por la iglesia, el estado, el ejército o la familia. Son, por tanto, altamente variables sociohistóricamente.

Para el ordenamiento interno del corpus establecí tres categorías de la dimensión poder: más poder $(+\mathrm{P})$, menos $(-\mathrm{P})$ e igual poder $(=\mathrm{P})$ que se cruzan con familia y con sociedad como puede observarse en la Tabla 1. Este cruzamiento dará como resultado seis tipos de situaciones comunicativas definitorias para la selección de las cartas que constituyen el corpus.

\begin{tabular}{|c|c|c|}
\hline & familia & sociedad \\
\hline más poder & $\mathrm{F}+\mathrm{P}$ & $\mathrm{S}+\mathrm{P}$ \\
\hline menos poder & $\mathrm{F}-\mathrm{P}$ & $\mathrm{S}-\mathrm{P}$ \\
\hline igual poder & $\mathrm{F}=\mathrm{P}$ & $\mathrm{S}=\mathrm{P}$ \\
\hline
\end{tabular}

Tabla 1 - Correlaciones posibles entre ámbitos sociales y poder

Establecí el grado de poder de acuerdo con el emisor de la carta. Los emisores $F+P$ (con más poder en el ámbito de la familia) podrían ser, por ejemplo, padres, abuelos, tíos, suegros; los emisores $S+P$ (con más poder en el ámbito de la sociedad) podrían ser, por ejemplo, jefes, empleadores, autoridades civiles o reales, los emisores F-P podrían ser, por ejemplo, hijos, hijastros, nietos, sobrinos, nueras, yernos, etc. De estas seis categorías $(\mathrm{F}+\mathrm{P}, \mathrm{F}-\mathrm{P}, \mathrm{F}=\mathrm{P}, \mathrm{S}+\mathrm{P}, \mathrm{S}=\mathrm{P}$ y $\mathrm{S}-\mathrm{P})$ solo cinco han podido ser bien documentadas, entendiendo por "bien documentada" un número considerable de textos entre los cuales poder seleccionar los que mejor cumplieran con las condiciones intra y extratextuales fijadas en nota $6^{11}$.

Otra cuestión teórica que conviene aclarar es el concepto de cortesía que utilizaré para el análisis de los datos. Universalmente, dirigirse al otro, supone el uso de estrategias de cortesia (Brown y Levinson 1987) que

11 Las características de la categoría $\mathrm{S}+\mathrm{P}$, y el contexto sociohistórico, explican las dificultades para su documentación dentro de los textos escogidos por los archivos. Las cartas que deben formar parte de esa categoría deberían ser aquellas en que un locutor con más poder se dirige a un alocutario con menos poder, no siendo estos, miembros de la misma familia. Tal es el caso de cartas de jefes, empleadores, autoridades civiles, reales, eclesiásticas, propietarios de comercios, estancias, etc. a empleados, subordinados, peones, changadores, esclavos etc. Para más detalles sobre este problema, ver Bertolotti (en prensa). 
forman parte del saber histórico, en el sentido de Coseriu (1981). Este saber histórico, según Koch (2008) se debe dividir en dos tipos de reglas manejadas por los hablantes: las reglas idiomáticas o propias de una lengua histórica particular, y las reglas discursivas, las de las tradiciones discursivas, en las cuales se inscriben, a mi juicio, las estrategias de cortesía, que explican, como veremos, algunos de los usos de las manifestaciones alocutivas.

Como ya lo señalara Labov hace ya más de tres décadas (1972: 827) existe una uniformidad en la lengua: los factores que operaron históricamente son los mismos que operan actualmente, lo cual no quiere decir que los parámetros y valores de estos sean idénticos en dos momentos diferentes de una "misma" sociedad. En este sentido, entiendo que algunas de las motivaciones de las elecciones de los hablantes pueden relacionarse con cuestiones psicopragmáticas tales como las estrategias de cortesía (Brown y Levinson 1987) en relación con distintos tipos de actos lingüísticos (Searle 1979).

Brown y Levinson (1987) consideran estrategias de cortesía las estrategias conversacionales utilizadas por el locutor para cuidar su propia imagen y la del y alocutario. Estos autores entienden que la imagen positiva pública es la que todos tratamos de mantener durante la interacción con otros individuos para obtener la aprobación de los demás. Conciben la imagen negativa como la necesidad que tenemos de que se respete nuestro espacio $y$ de que no nos dirija en nuestro accionar. Estos autores proponen que, para mantener la necesaria cortesía social, las amenazas lingüísticas a ambos tipos de imagen deben ser reparadas. Llaman entonces cortesía positiva a las estrategias lingüísticas que apuntan a la conservación de la imagen positiva y cortesía negativa a las que buscan atenuar la eventual afectación a la imagen negativa. Las estrategias de cortesía se asocian, entonces, con la realización de los actos que amenazan la imagen (Brown y Levinson, 1987: 61).

Para generalizar los actos lingüísticos que pueden amenazar la imagen del alocutario, me sirvo aquí del concepto de actos directivos, unos de los cinco definidos por Searle (1979: 29) cuya caracterización presento ligeramente adaptada: asertivos: a través de ellos le decimos a la gente cómo son las cosas; directivos: a través de los cuales intentamos lograr que los otros piensen o hagan cosas; compromisivos: a través de los cuales nos comprometemos a hacer cosas; expresivos: a través de los cuales expresamos nuestros sentimientos, pensamientos y actitudes y declarativos: a través de los cuales producimos cambios con nuestras emisiones.

En cuanto a aspectos metodológicos del análisis, cabe aclarar que si bien este es un estudio cualitativo, en el entendido de que la frecuencia de uso en diversos contextos puede ser indicador de la institucionalización 
de nuevas rutinas lingüísticas (García, 1999, 2004), realizo algunos conteos básicos sin pretensión estadística.

$\mathrm{El}$ análisis que retomo parcialmente en este trabajo fue realizado en Bertolotti (en prensa) - análisis de la presencia de sujetos pronominales alocutuivos singulares - y en Bertolotti (2009) - análisis de las manifestaciones del objeto directo alocutivo -. Siguiendo la tradición de Brown y Gilman (1960) uso la notación $t$ vs. $v$ para establecer la oposición cercanía vs. distancia

En primer lugar, realicé el relevamiento de la totalidad de ocurrencias de los contextos de distancia o contextos- $v$. Consideré si los verbos- $v$ tenían o no un sujeto explícito y relevé la totalidad de las ocurrencias en las que había un objeto directo-v, distinguiendo si se trataba del primer o el segundo período bajo estudio.

Luego, puse en relación las ocurrencias con los ámbitos de sociales y con el poder de los locutores, para comparar lo que sucedía en iguales ámbitos en el primer y en el segundo período.

Realicé exactamente el mismo procedimiento con las formas-t, es decir, de formas de cercanía, ya que me interesaba saber qué comportamientos eran compartidos y cuáles no por los miembros de la clase de los pronombres personales alocutivos sujeto y objeto directo en el español del siglo XIX en Uruguay.

\section{Los datos y su análisis}

\subsection{Los datos sobre el sujeto}

\subsubsection{Sujetos- $\nu$ en siglo $\mathrm{XIX}_{1}$ y siglo $\mathrm{XIX}_{2}$}

El análisis de la totalidad de las ocurrencias del corpus en las que un verbo-v presenta explícito un sujeto usted, presentado en Bertolotti (en prensa) me permitió concluir que la presencia de usted tiene tres posibles razones en el español del siglo XIX en Uruguay: a) la identificación (desambiguación, contraste, énfasis), b) la generación de contextos de complicidad o c) la cortesía o la referencialidad, razones que desarrollo y ejemplifico a continuación. Más adelante, presentaré el análisis cuantitativo realizado. 
Los sujetos usted cuya presencia entiendo que está al servicio de la identificación se presentan en contextos en los que hay posible ambigüedad en la interpretación, se quiere contrastar con otro participante o se quiere realizar algún tipo de énfasis en el alocutario.

En el ejemplo (1), en la línea 24, la escritora evita una posible interpretación incorrecta de su frase ya que, entre la última expresión en referencia al alocutario (Reciba usted, en la línea 23) y el nuevo uso del verbo recibir dirigido al alocutario, se interpone un nombre propio Marcelina que podría también ser un buen candidato a sujeto de recibe, de la línea 25.

(1) [fol. 1]

Reciva V. expreciones de Marcelina la q. ignora aun el modo como se las ha dedar, pues no es facil adivinar como las recive V. Mil afetos de todos y en

25. particular de su invariable servidora

$$
\begin{aligned}
& \text { q. B.S.M. } \\
& \text { A. Collis }
\end{aligned}
$$

236. Carta de Andrea Collis a Juan Ponce

Otro ejemplo de usted sujeto explícito identificador puede verse en el ejemplo (2) en el cual el pronombre contrasta con otro referente humano inmediatamente anterior (Juan Pedrito) y sirve de apoyo a la aposición mi querido y amado primo.

(2) $[\because$ fol. $1 \mathrm{v}]$

A Juan Pedrito mil cariñosos abrasos

y vm mi querido y amado Primo cuente

15. sienpre con el agradecido afecto de su amante

prima q.e desea con su corason ber avm y abra

sarlo quanto antes

182. Carta de una prima a Cristóbal Salvañach

4.1.1.1.b) la generación de contextos de complicidad

Este uso se caracteriza, a mi juicio, por desencadenar efectos de verdad sobre la proposición subordinada y, establecer, de esta manera, un mundo compartido entre locutor y alocutario. Esta construcción pa- 
rece ser más frecuente con sujetos-t, por lo tanto, me explayo algo más sobre la cuestión en 4.1.2.

(3) [*fol. 1r]

17. la carta que ledririjo con su Ayte. Iglesias, le aseguraría esta verdad; en ella verá V. que le hablo con la franqueza que V. ya conoce enmí, en el bien entendido que mi tendencia es el Pais, nada más

239. Carta de Eugenio Garzón a Fructuoso Rivera

(4) $[$ fol. $64 \mathrm{v}]$

13. amigo la letra bá desfigurada: vd. mande á este su amigo q. ${ }^{e}$ vd. ya sabe quienes

237. Carta anónima a José Artigas

En (3) Eugenio Garzón “obliga” a Rivera a asumir que Garzón es franco. En (4) al autor anónimo, al tiempo que desambigua el sujeto de sabe lleva a Artigas a aceptar el conocimiento de su identidad.

\subsubsection{1.c) la cortesía}

Todos los sujetos explícitos cuya presencia no se explica ni por la necesidad de identificación ni por la estrategia de construir un mundo compartido se usan, a mi juicio, por razones relacionadas con la cortesía.

Al ser "innecesaria" la aparición de estos pronombres, su uso debe tener una justificación el hablante debe estar "perfilando" la realidad de alguna manera. En (5) nos encontramos frente a un usted sujeto explícito que acompaña a un verbo en imperativo, la flexión verbal que pude prescindir de sujeto explícito por excelencia. En (6) usted es sujeto de un futuro de mandato en un contexto no ambiguo, ya que no hay otro participante. En ambos casos, usted es prescindible. ¿Por qué los escritores eligen ponerlo, entonces? Creo que la presencia de usted habilita una interpretación más amable, más deferente, más cortés del imperativo o del futuro con sentido de mandato.

(5) [*fol. 1r] Venda Vd. cuanto tenga para asistirla que es lo primero, y atender á mi querido José María que para eso he trabajado

230. Carta de José Artigas a su suegra Francisca de Villagrán 
(6) [*fol. 1v]

15. rrecivira Vmd. memorias de.

Maria laquese álla mui alentada y dicetiene

Ganas deasèr otro hijo

27. Carta de Juan García a su padre

El análisis del conjunto de los ejemplos mostró que este uso "innecesario", que interpreto como cortés, suele aparecer en general en contextos de actos directivos (Bertolotti en prensa). Los actos directivos pueden ser considerados actos de amenaza a la imagen del alocutario, y el uso de usted sería entonces una estrategia de cortesía para atenuar la amenaza a la imagen del alocutario. Entiendo, entonces, que la presencia de usted "innecesarios" asociados a actos directivos, puede explicarse como una estrategia de cortesía hacia el alocutario a quien se le está pidiendo u ordenando algo.

El análisis de los ámbitos de comunicación (familia o sociedad) y de las relaciones de poder (mayor, igual o menos poder) nos muestra que la mayor concentración de sujetos usted explícito se presenta en ámbitos sociales en los que el locutor tiene menos poder que el alocutario, seguida de ámbitos sociales e igual poder en el ámbito social y luego por frases producidas en el ámbito familiar por escritores de menor poder. No se presentan usos de usted explícito en situaciones en la que el locutor tiene más poder que el alocutario. Estos resultados se pueden observar en la Tabla 2:

\begin{tabular}{|l|l|l|}
\hline & \multicolumn{2}{|c|}{ sujetos-v } \\
\hline $\mathrm{F}+\mathrm{P}$ & - & $0 \%$ \\
\hline $\mathrm{F}=\mathrm{P}$ & 4 & $4.5 \%$ \\
\hline $\mathrm{F}-\mathrm{P}$ & $\mathbf{6}$ & $\mathbf{8 . 9 \%}$ \\
\hline $\mathrm{S}=\mathrm{P}$ & 19 & $28.3 \%$ \\
\hline $\mathrm{S}-\mathrm{P}$ & $\mathbf{3 9}$ & $\mathbf{5 8 . 2} \%$ \\
\hline & 67 & $99.9 \%$ \\
\hline
\end{tabular}

Tabla 2 - Sujeto explícito usted de acuerdo con el ámbito siglo XIX

De la lectura de la Tabla 2, surge la existencia de una correlación clara entre menos poder y ámbitos no familiares, es decir, las situaciones en las que el uso de estrategias de cortesía es más necesario presentan un uso de usted sujeto explícito más frecuente. 
El análisis pormenorizado de las situaciones $\mathrm{S}=\mathrm{P}$, en las que el locutor detenta igual poder que su alocutario, arroja resultados interesantes. De los 19 usted sujeto explícito en contexto $\mathrm{S}=\mathrm{P}, 17$ se encuentran en contextos de actos directivos, más específicamente de mandato (más o menos real): 11 de esos actos directivos están expresados en imperativo y 6 en subjuntivo con significado de mandato o con perífrasis con sentido obligativo.

En el ejemplo (7) es posible observar diferentes variantes de esta situación: usted como sujeto de imperativo (línea 23), usted como sujeto de perífrasis con sentido de obligación (línea 13 y línea 30) y usted como sujeto de un verbo en subjuntivo presente regido por un verbo de obligación (línea 15).

(7) [fol. 1]

Suplico a V. se tome la molestia de decirle al cavallero Vallan q. ${ }^{e}$ Zermiño

10. me participa le ha entregado vna pza de gaza ([de]) azul q. ${ }^{e}$ volverá a su poder sino varía de parecer; pues ami me dixo me la daria en seys pesos, y hahora salimos con q. ${ }^{e}$ han de ser siete con seys reales; a ese precio no puedo recivirla yla devolvere con la vrevedad posible. No dexe $\mathbf{V}$. de abisar la resolución de este cavallero sobre el particular, como asi mismo me ha vuscado lacaxa de rapé q. ${ }^{\text {e }}$

15. hencomendé me la vusque V. la entregue en casa, para que el conductor de esta me la trahiga.

(...)

Reciva V. expreciones de Marcelina la q. ignora aun el modo como se las ha dedar, pues no es facil adivinar como las recive V. Mil afetos de todos y en

25. particular de su invariable servidora

$$
\text { q. B.S.M. }
$$

A. Collis

P.D.

30. La adjunta sirvase V. entregarla al Sor Medina y exigir contestacion conla vrevedad posible pues deseo la trahiga el conductor de la presente.

236. Carta de Andrea Collis a Juan Ponce 
El análisis cuantitativo y cualitativo hecho para el siglo XIX Xue $_{1}$ acabo de resumir fue realizado también en el corpus del siglo XIX. Los resultados cuantitativos evidencian dos cuestiones: a) que el número de ocurrencias de usted disminuye notoriamente (63 en Siglo XIX frente a 32 en siglo $\mathrm{XIX}_{2}$ ) y b) que ha cambiado la distribución de acuerdo con el ámbito de relacionamiento en que los interlocutores se expresan. Ambas cuestiones pueden apreciarse en la Tabla 3:

\begin{tabular}{|l|l|l|}
\hline & \multicolumn{2}{|c|}{ sujetos-V } \\
\hline $\mathrm{F}+\mathrm{P}$ & $3^{12}$ & $8.6 \%$ \\
\hline $\mathrm{F}=\mathrm{P}$ & - & - \\
\hline $\mathrm{F}-\mathrm{P}$ & 12 & $34.3 \%$ \\
\hline $\mathrm{S}=\mathrm{P}$ & 12 & $34.3 \%$ \\
\hline $\mathrm{S}-\mathrm{P}$ & 8 & $22.8 \%$ \\
\hline & 35 & $100 \%$ \\
\hline
\end{tabular}

Tabla 3 - Sujeto explícito usted de acuerdo con el ámbito siglo XIX ${ }_{2}$

Si bien los tres ámbitos en que se encuentran los pronombres usted sujeto mayoritariamente siguen siendo los mismos que en el siglo $\mathrm{XIX}_{1}$, las construcciones con sujeto explícito no se concentran mayoritariamente en S-P $(58 \%$ en S-P en el siglo XIX frente a $22.8 \%$ en S-P en el siglo $\left.\mathrm{XIX}_{2}\right)$.

El análisis realizado en Bertolotti (en prensa) muestra que la disminución porcentual y la desconcentración en el ámbito S-P se asocia con la disminución de usted sujeto explícito como estrategia de cortesía, de reparación a la amenaza a la imagen del alocutario. También disminuyen las interpretaciones corteses en los contextos F-P.

Los únicos casos en que cabe una interpretación ligada a deferencialidad se encuentran en dos cartas, ambas de sobrinos a tíos. Ejemplifico con una de ellas (ejemplo 8) que cuenta, además, con usos de pronombres sujeto explícito al servicio del énfasis y del contraste. El

12 Puede llamar al atención que aparezcan formas- $\nu$ en un contexto F+P. En este caso las tres formas en cuestión están en un texto en el que el padre trata de usted a su hijo con un sentido estilístico; cambia a esta forma en medio de una carta- $t$ cuando comienza a tratar al niño de igual a igual; trata al niño como a un adulto. 
locutor, Juan Luis Blanes es un escritor culto $^{13}$, que está estudiando arte en Florencia y desde allí escribe su carta, no exenta de cierta ceremoniosidad que no condice con la relación cercana a la que aluden datos biográficos.

(8) [*fol. 1r]

1.

Florencia Julio 5/ 879

Querido tio.

Con fecha 18 de Mayo $\mathrm{pp}^{\circ}$

5. se ha recibido en casa una carta de $\mathrm{Vd}$ por la cual vemos que sigue bien de salud á Dios gracias; _en dicha carta, papá me hizo leer la parte que se refiere al tal Urta y con

10. cuyo motivo dice Vd:

(...)

25. El primero del pasado mes escribí á Vd dandole cuenta de nuestro viage; me hará $\mathbf{V d}$ el servicio de avisarle a $\mathrm{Da}$ Cris $=$

= tina y a las muchachas [??]amas

30. que tambien á ellas les he escrito.

[*fol. 1v]

Deberá saber Vd que desde el

7 de Junio he ingresado a la $\mathrm{A}=$

=cademia donde concluyo un

hermoso carton que pronto irá

5. á esa

Adjunto diarios para que

Vd se entere del contenido

(...)

Mis espresiones a las

muchachas [??]amas, Tarda $=$

20. -guila y el Sr Masariego, y Vd

reciba un abrazo de su sobri=

13 En el sentido que da a este término Oesterreicher (1996: 324-325), por oposición a semiculto, es decir, quienes "escriben o dictan un texto sin conocer suficientemente ni la variedad lingüística exigida por el género respectivo ni las reglas discursivas válidas para la estructuración del texto, y que, muchas veces, no saben aprovechar las posibilidades de la comunicación escrita." 


\section{BERTOLOTTI, Virginia.}

$$
\begin{aligned}
& =\text { no que lo quiere } \\
& \text { Juan Luis Blanes }\{R U B\}
\end{aligned}
$$

131. Carta de Juan Luis Blanes a su tío Mauricio

En el siglo XIX, la mayor parte de las ocurrencias del sujeto usted explícito pueden explicarse como casos de identificación, sobre todo de énfasis. En los contextos $\mathrm{S}=\mathrm{P}$ en el siglo $\mathrm{XIX}$, los usos de usted sujeto explícito son predominantemente enfáticos, contrastivos, o sea, de identificación.

Una de las excepciones dentro del ámbito $\mathrm{S}=\mathrm{P}$ es similar a la que habíamos visto en el (ejemplo 8), y aparece en una carta de un intelectual a otro en la que le agradece el envío de bibliografía (ejemplo 9).

(9) [*fol. 1r]

$$
\text { No se imagina }
$$

Ud. con cuanto empeño había

20. buscado desde hace años las

Noticias estadísticas, cuya in

teresante introduccion me en-

[*fol. 2r]

1. vía Ud.

266. Carta de Carlos María de Pena a Andrés Lamas

En síntesis, el análisis de las razones semánticas y pragmáticas de aparición del sujeto explícito usted en situaciones F-P, S-P, S=P en el período siglo $\mathrm{XIX}_{2}$ son las mismas que en el anterior, pero en proporciones marcadamente distintas. Aparece fundamentalmente con un sentido de identificador, ya para establecer contraste con otros participantes, ya para hacer énfasis en el alocutario, o simplemente para desambiguar. El uso que he llamado cortés se presenta excepcionalmente. Entre esas ocurrencias excepcionales consigné su uso en escritores cultos, que aparentemente son capaces de rutinas lingüísticas más conservadoras, seguramente, porque conocen mejor las tradiciones discursivas.

Esto contrasta fuertemente con lo que sucedía en el siglo XIX período en el cual el uso cortés era fuertemente mayoritario en las situaciones S-P y también estaba presente en las situaciones $\mathrm{S}=\mathrm{P}$ y F-P. 


\subsubsection{Sujetos-t en siglo $\mathrm{XIX}_{1}$ y siglo $\mathrm{XIX}_{2}$}

Las razones que justifican los usos explícitos de tú y de vos son primordialmente: a) la identificación (desambiguación, contraste) y b) la generación de contextos de complicidad. A diferencia de lo que sucede con los sujetos usted ${ }^{14}$, son casi inexistentes los sujetos- $t$ corteses.

Los sujetos-t aparecen mayoritariamente en contextos de identificación. Dentro de la identificación, los usos contrastivos son los más abundantes. Ejemplifico con dos ocurrencias (ejemplos 10 y 11): en la línea 18 de (10) tú contrasta con hermano. En la línea 6 de (11) tú contrasta con el propio locutor pero sobre todo con la compañia de Gias.

$$
\text { me pondras alos }
$$

pies de ermano y tu reciviras los= afectos de todas. particularmte de

20. tu ermana q.e te ama

Carmen Fragoso $\{$ RUB $\}$

245. Carta de Carmen Fragoso a su hermana Bernardina

(11) [*fol. 1]

5.

en fin por hora venga pronto la compañia de Gias y tu vendras cuando yo vaya. amigo es preciso abrirlos. esta familia no corre muy vien y por lo mismo es preciso que no todo sea amen, amen, como ellos quieren para aser sera y pavilo del pais y de nosotros.

225. Carta de Fructuoso Rivera a su hermano Bernabé

La fuerza del contraste es tal que incluso permite prescindir del verbo como se puede leer en otros documentos: Dale a Federico mis cariñosas expresiones y toda esa querida familia y tú un abrazo de tu mamá (Documento 275, folio $2 \mathrm{v}$, líneas 16 a 19).

Como ya señalé, también se encuentran sujetos-t explícitos en contextos de complicidad, que se caracterizan por la co-ocurrencia de las formas de sujeto explícito con verbos del tipo semántico de saber (conocer, comprender, etc.).

14 He registrado algún uso cortés con vos en cuyo análisis no puedo profundizar aquí. 
Esa construcción desencadena la presuposición de verdad del contenido de la completiva. El locutor obliga así al alocutario a compartir sus opiniones, a entrar en su mundo. Si en lugar de "decir" tú sabes los locutores solo escribieran sabes, el alocutario podría esperar una pregunta y no una declaración. El tú le da asertividad a la construcción.

Comparemos (a) Tú sabes que X con (b) Sabes que X. En (a) el lector interpreta que la completiva "que X" es verdadera, en tanto que en (b) el lector podrá hacer la misma interpretación que (a), pero también puede esperar que la completiva que sigue a sabes sea una interrogación sobre el conocimiento del contenido de la completiva, con entonación interrogativa. La forma tú - y la ausencia de entonación interrogativa -, entonces, le quitan responsabilidad al locutor y se la dan al alocutario. Tú sabes no solo significa que el alocutario sabe sino también que el locutor asume que el alocutario sabe $\mathrm{X}$ y lo arrastra en esta asunción. Es la presencia de tú que hace al alocutario responsable de la existencia del enunciado del locutor. Ante la presencia del pronombre, entonces, el alocutario debe interpretar al expresión tú sabes como parto de la base de que compartimos $X$ información.

Tal como en el caso de los sujetos- $v$, en Bertolotti (en prensa) repliqué

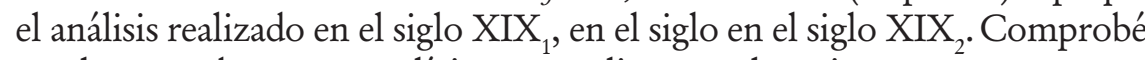
que los usos de tú o vos explícitos se explican por las mismas razones que en el siglo $\mathrm{XIX}_{1}$, dividiéndose entre la identificación - con cierta preferencia por usos enfáticos - y los usos de complicidad. A diferencia de lo que sucede con usted sujeto explícito, las formas-t no presentan diferencias cuantitativas sustanciales con respecto al período anterior.

En síntesis, el análisis realizado revela que la presencia de usted es cualitativamente diferente que la presencia de tú o vos sujeto explícito en ambos períodos, ya que los usos corteses se asocian contundentemente a usted. Cuantitativamente, la presencia de usted sujeto explícito es mayor en el primer período bajo análisis que en el segundo y es, en ambos períodos, superior a la de tú o vos $^{15}$.

\subsection{Los datos sobre el objeto directo}

El análisis del corpus evidencia que la expresión del alocutario singular como objeto directo, es decir, como argumento adyacente directamente al verbo, asume cuatro manifestaciones: $\theta$, pronombre átono acusativo, preposición más su término pronominal o los dos últimos recursos

15 El número de ocurrencias de vos es menor que el de tú, de allí que haya decido ejemplificar con tú. 
simultáneamente: pronombre átono acusativo y preposición más su término pronominal. Estas últimas construcciones son llamadas por Fernández Soriano (1999: 1248) casos de "duplicación propiamente dicha".

Analizaré en esta ocasión la función sintáctica objeto directo en las siguientes tres manifestaciones pronominales ${ }^{16}:$ a) [pronombre átono acusativo + verbo]: tello la saludo; b) [verbo $+\mathrm{a}+$ pronombre tónico]: saludo a ti a vos/ a usted; c) [pronombre átono acusativo + verbo $+\mathrm{a}+$ pronombre tónico]: te/ lo la saludo a ti a vos/ a usted.

En cuanto a sus funciones semánticas, "el tipo de participación en el evento de los objetos directos varía notoriamente según el verbo del que se trate, a lo sumo puede constatarse una correlación negativa con el papel agente o causa, que difícilmente puede asociarse con un objeto directo" (Laca, 2006: 424)

El análisis que realicé sobre el objeto directo (Bertolotti, 2009) incluye menos variables que el realizado sobre el sujeto, ya que el número de ocurrencias es sensiblemente menor y aumentar el número de variables me hubiera llevado a la inviabilidad del análisis cuantitativo. Por lo tanto, voy a contrastar formas- $t$ vs. formas- $v$ en los dos períodos bajo estudio, siglo $\mathrm{XIX}_{1}$ y siglo $\mathrm{XIX}_{2}$, sin considerar cuantitativamente los ámbitos de uso o las relaciones de poder entre los participantes.

\subsubsection{Objetos directos-t $\mathrm{y}-\mathrm{v}$ en siglo $\mathrm{XIX}_{1}$}

Como puede observarse en la Tabla 4, en el siglo XIX , la expresión del objeto directo en contextos-t, es decir, en contextos de cercanía, se da mayoritariamente a través del pronombre átono acusativo te (29 casos). No hay casos de objeto directo preposicional ni duplicación de pronombre átono acusativos con frase preposicional.

\begin{tabular}{|l|c|c|c|c|c|c|c|c|}
\hline \multicolumn{10}{|c|}{ formas- $t$} & formas- $\nu$ \\
\hline & te & $a$ ti $\sim \operatorname{vos}$ & 0 & doble $e^{17}$ & lo/la & $a$ usted & 0 & doble \\
\hline siglo XIX & 29 & - & 2 & - & 9 & 8 & - & 2 \\
\hline siglo XIX & 29 & 4 & - & 3 & 14 & 4 & 1 & 2 \\
\hline
\end{tabular}

Tabla 4 - Expresión del objeto directo en siglo XIX 1 y siglo XIX

16 No entiendo conveniente incluir en este análisis los casos, excepcionales, de ausencia de marca.

17 Se forma con ocurrencias de pronombre átono y preposición más pronombre tónico, contabilizadas en sus respectivas columnas. 
En (12) se puede apreciar un ejemplo de expresión de objeto directo a través del pronombre átono acusativo

(12) [*fol. 2r]
turresibiras el
20. fino cariño de tu fiel espos $\mathrm{q}^{\mathrm{e}}$ tes
tima y berte desea,

30. Carta de José de Mesa a su esposa

Las estrategias de los escritores para la expresión del objeto directo en el siglo $\mathrm{XIX}_{1}$ son radicalmente distintas en contextos- $v$. Como se puede constatar en la Tabla 4, 7 de los 15 casos de verbo con objeto directo encontrados $(47 \%)$ se expresan exclusivamente a través de pronombre átono acusativo (contra 100\% de pronombre átono acusativos-t) en tanto $6(32 \%)$ se expresan a través de "preposición + usted" (contra $0 \%$ de preposición + pronombre-t). El 21\% restante está compuesto por duplicaciones, es decir, el uso simultáneo de ambas estrategias de expresión, [(pronombre átono acusativo) + verbo + (preposición + pronombre tónico)], construcción que no se presenta en el ámbito-t-

De acuerdo con la descripción gramatical del siglo XX (ver por ejemplo, Fernández Soriano $\$$ 19.4.1.) la duplicación es obligatoria cuando el objeto canónico es un pronombre (Me ha visto a mí, Nos llamó a nosotros), pero no lo es en el caso de usted. No obstante, la no duplicación ocurre 6 veces en mi corpus del siglo $\mathrm{XIX}_{1}$ (sobre un total de 15 objetos directos) y 2 veces en mi corpus del siglo XIX (sobre un total de 16 objetos directos). En (13) la sintaxis obliga a la aparición de usted porque la elisión de verbo sería imposible sin el anclaje que permite el pronombre; por otra parte, hay un nombre en aposición (compadre) (folio 1r línea 11) que permite ligar la frase con otra nueva, también con el verbo reconocer elidido.

[fol. 1r]

toda la persona en la lista de esta nose presiente a esta reunion Gra?l serreconosera por

10. enemigo y contrario al exercito. de la libertad y así nosolamente a Vsted conpadre sinos atodos los que Vsted pueda dar parte de que ciga este mismo orden es todo quanto se me ofrese darle parte 
El resto de los usos de objeto directo preposicional con usted pueden interpretarse como guiados por la necesidad semántica de asegurar la interpretación humana del objeto directo. Como ha señalado Aissen (2000 apud Laca (2006: 436) dos escalas pueden caracterizar la marcación de los objetos directos: la de la animación (humano $>$ animado $>$ inanimado) y la de la definitud (pronombre ${ }^{18}>$ nombre propio $>$ definido $>$ indefinido $>$ indefinido específico $>$ no específico).

Si consideramos que el pronombre átono acusativo, por cierto, recubre cualquier signficado, ocupe el lugar que ocupe en esa escala y que un alocutario, por ser humano, se ubica en el punto primero de ambas escalas, puede postularse un conflicto entre el hecho de que lo/la recubra la totalidad de las escalas y la necesidad de los hablantes de poner de manifiesto el carácter animado del alocutario.

Entiendo que el uso del pronombre átono acusativo no relevaría suficientemente la (evidente) condición de humano del alocutario. Esto es, si el alocutario es humano y por lo tanto ocupa el primero de los lugares en la escala de animación y se lo expresa por el pronombre átono acusativo lo se está usando una manifestación que ocupa también el tercer lugar en la escala de animación y el sexto en la de definitud. Sin embargo, el uso de usted en lugar de los posibles lo/la empareja un significado de primer lugar en la escala de animación con una forma del primer lugar en la escala de definitud. Esta elección de los hablantes satisface las necesidades expresivas pero tiene un sesgo "aparadigmático" ya que se usan formas tónicas para la expresión de una función sintáctica en la que otras subclases del paradigma usan formas átonas.

Por ejemplo, en (14), y pasando por alto su carácter formulaico, el uso con lo sin expresión que refuerce su característica de humano podría permitir la interpretación del verbo guardar como "conservar algo dentro de otra cosa" (Dios lo guarde y lo mantenga guardado muchos años) y no como "cuidar a alguien".

(14) $[*$ fol 1v]

1. A Vmd. reconsido y Con esto

D. s. g. á Vmd m. años para que

Mande a su mas atento Serbidor

q. ${ }^{e}$ sus Manos B. ${ }^{a}$ en Casupa

18 Entiendo que con pronombre refiere al pronombre sujeto. 
$5 \quad$ A 27 de Julio de 1793 queda de Vmd Spre

Josef Antonio Artigas

de

240. Carta de José Antonio Artigas a Juan Francisco García

Al continuar analizando las expresiones de objeto directo, se hace evidente, como es posible observar en los ejemplos (15) a (18), que las expresiones con usted tienen en común no tener el papel semántico de paciente que es el que prototípicamente tiene el objeto directo: romper [algo $]_{\text {tema }}$ Se trata de entidades animadas, que son desde el punto de vista semántico, malefactarios (ejemplos 15 y 16) o cercanos a un beneficiario (ejemplos 17 y 18).

(15)

9. es todo quanto se me ofrese dar parte y enfadar a Vmd. quien de todo corason

desea cerbir

234. Carta de Manuel Vega a José Oserbante

(16) [fol. 64r]

1.

Montevideo Marzo 8 de1811

S. ${ }^{r}$ D. ${ }^{n}$ JosepArtig.s muy S.r mío, no extrañe Vd. el que yo pase á yncomodár á vd.

237. Carta anónima a José Artigas

(17) $[*$ fol. 1v]

vm mi querido y amado Primo cuente

15. sienpre con el agradecido afecto de su amante

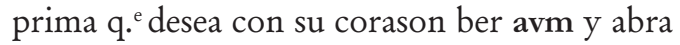
sarlo quanto antes

182. Carta de una prima a Cristóbal Salvañach 
(18) [*fol. 1r]

1. Sor Dn Andrés Lamas

Mi respectable Sor y amigo

5. esta se dirije despues de saludar á V.

para $q^{e}$ me haga la gracia de emprestarme

media docena de pesos pues V. no debe [??]

hignorar como habremos venido de ca["mpa]

243. Carta de Bernabé Rivera a Andrés Lamas

Además, algunos de estos usos se dan en contextos de expresión cortés positiva, lo que podría también obligar a un grado más alto de personalización que el que ofrecería el pronombre átono acusativo lo o la.

\subsubsection{Objetos directos-t $\mathrm{y}-\mathrm{v}$ en el siglo XIX2}

En el siglo $\mathrm{XIX}_{2}$ la situación para las formas-t se distingue de la del período anterior en que se registra un caso de objeto directo preposicional pronominal "preposición + vos" y dos casos de duplicación: uno con vos y uno con ti, en ambos casos de intención enfática.

El caso de "preposición + vos" (ver ejemplo 19) se explica por la necesidad de coordinar con un sintagma nominal (demás familia).

\footnotetext{
20. mano. Me es grado saludar

a voz y demas familia con $\mathrm{mi}$

mayor aprecio S.S.
}

256. Carta de Francisco Saravia a su hermano Aparicio

El comportamiento de las formas- $v$ cambia considerablemente. El número de expresiones con usted en el objeto directo desciende proporcionalmente. Pasa de 8 en 17 a 4 en 14 o sea de un $43 \%$ a un $28 \%$. De esas tres, solo uno está duplicado y otro aparece sin la preposición. En los cuatro casos se trata de objetos directos del verbo saludar, tal como se 
mostró en ejemplo anterior. La primera explicación a esto podría ser que se trata de una rutina discursiva en la que se fijó formulaicamente con el verbo saludar la posibilidad de un objeto directo con pronombre tónico sin expresión duplicada del pronombre átono acusativo. Esta rutina compite, sin embargo, con otra que usa el pronombre átono acusativo: se cuentan en el corpus los mismos casos con el pronombre átono acusativo lo o la. Comparé los contextos en que aparecen, para tratar de inferir si hubo alguna estrategia por parte de los locutores(ver Cuadro 1).

\begin{tabular}{|c|c|c|c|}
\hline \multicolumn{2}{|r|}{ preposición + usted } & \multicolumn{2}{|r|}{ pronombre átono acusativo } \\
\hline a. & $\begin{array}{l}\text { Respetable } \mathrm{S}^{\mathrm{r}} \text { despues de saludarlo á } \\
\text { Uste paso á molestar su dinida pidien- } \\
\text { dole me haga el serbicio de soltar á mi } \\
\text { hijo Anjel Caseres (Doc. 196) }\end{array}$ & e. & $\begin{array}{l}\text { Estimado compadre pariente y amigo: } \\
\text { esta tiene por objeto el saludarlo, y al } \\
\text { mismo tiempo decirle que desde el } \\
\text { principio de este mes le escrito tres } \\
\text { cartas (Doc. } 164 \text { ) }\end{array}$ \\
\hline b. & $\begin{array}{l}\text { no pude pasar asta esa con este motibo } \\
\text { mes grato saludar a } \mathbf{V} \text { en conpaña de } \underline{s u} \\
\text { estimada familia que dios guarde } \\
\text { muchos años-de V Aftismo SS y amigo } \\
\text { (Doc. } 254)\end{array}$ & f. & $\begin{array}{l}\text { Estimado compadre y pariente: esta } \\
\text { tiene por objeto el saludarlo desendole } \\
\text { saluz y felicidades y almismo tiempo } \\
\text { decirtle (Doc. } 165)\end{array}$ \\
\hline c. & $\begin{array}{l}\text { muy señor mio y amigo des pues } \\
\text { desaludar a Vdcon el mayor rrespeto } \\
\text { paso à decirle, puesci es cierto que el } \\
\text { departamento Durasno es nuetro pido } \\
\text { abuese } n_{\text {i }} \text { cia la comisaria (Doc. } 264 \text { ) }\end{array}$ & g. & $\begin{array}{l}\text { Confiando en que la distinguida } \\
\text { Señora aceptara lo que ofresco. la } \\
\text { saluda con su consideración más } \\
\text { distinguida (Doc. 200) }\end{array}$ \\
\hline d. & $\begin{array}{l}\text { Querido Tío: La presente tiene por } \\
\text { objeto saludar á Ud y demás familia } \\
\text { (Doc. } 270 \text { ) }\end{array}$ & h. & $\begin{array}{l}\text { cinl otro par ticular le saluda con el } \\
\text { rrespeto que me es debido rrepitien- } \\
\text { dome de V yfamilia su afmo SS (Doc. } \\
\text { 264) }\end{array}$ \\
\hline
\end{tabular}

Cuadro 1 - Comparación de ocurrencias del verbo saludar con pronombre tónico y átono.

El análisis sugiere que más allá de que saludar es una expresión léxica de cortesía positiva en sí misma, en los tres primeros casos de la columna (sin duplicación y con usted ( $\mathrm{a}, \mathrm{b}$ y $\mathrm{c}$ ) se trata de pedidos en situaciones S-P y en el último (d) se trata de una situación F-P. En este último caso la sintaxis también justifica la presencia de a usted lo cual no sucede en los tres anteriores. El autor no podría haber dicho * ${ }^{*}$ aludarlo y demás familia, pues no es posible coordinar un sintagma nominal con un pronombre átono acusativo. 
En los dos primeros casos de la columna pronombre átono acusativo (e y f) se trata de situaciones $S=P$. En ambos textos, inmediatamente después de saludar, el locutor pasa a tratar cuestiones comerciales. Distinto es el caso de los dos segundos ( $\mathrm{g}$ y h) en los que la situación es S-P. En ellos, luego del pronombre átono acusativo, hay sendas expresiones léxicas de cortesía (con su consideración más distinguida en uno y con el respeto que me es debido en otro), y el locutor se distancia a través de la tercera persona (la saluda; le saluda). En el primero de los casos viene de nombrarla como la distinguida señora. La apelación a manifestaciones de cortesía positiva en $(\mathrm{g})$ y $(\mathrm{h})$, estaría quizás sustituyendo lo que en los ejemplos de la primera columna se lograba con la explicitación de usted. ${ }^{19}$

Esto me lleva a pensar que la elección de la construcción con usted es una apuesta del locutor a la cortesía positiva a través de la mayor materialidad de esta forma, a lo que se suma la persistencia de los valores de usted, lo cual discutiré a continuación.

En síntesis, tal como sucede con el sujeto, formas- $t$ y formas- $v$ objeto directo presentan comportamientos dispares. Esta diferencia se centra fundamentalmente en la expresión objetiva a través de [verbo $+a+$ pronombre tónico] que es una opción utilizada en casi la mitad de los casos en las formas de lejanía y apenas usada en las formas de cercanía. Estas manifestaciones de a usted disminuyen a lo largo del siglo, al tiempo que aumentan las de los pronombre átono acusativos to o la.

Mi hipótesis es que la mayor explicitud de a usted que la del clítico se relaciona con una estrategia discursiva para evidenciar el carácter humano del alocutario. Infiero que para los hablantes de la época, el uso del pronombre átono acusativo podría resultar opaco, dado que esta codifica objetos con otras características semánticas. Esta opacidad sería consecuencia de la sistematización que llevó a que las concordancias de vuestra merced se fueran consolidando en la tercera persona. Como se mostró, la opción por la forma $a+$ usted se refuerza, además, en contextos de cortesía.

19 Como se vio más arriba, además, el caso del verbo saludar, se inscribe en ese eventual desfasaje de escalas ya que su segundo argumento es un objeto directo (y en tanto tal se codifica a través del pronombre átono acusativo $/ 0 /$ a) a pesar de ser el referente humano. 


\subsection{Discusión de los datos ${ }^{20}$}

El análisis realizado permite constatar cambios en los usos de los sujetos y objetos del paradigma de usted. En ambos casos se constata una disminución de las manifestaciones que incluyen el tónico usted. Este hecho es exclusivo de esta subclase y la diferencia de las formas-t.

He argumentado que la presencia de formas- $v$ en contextos en los que hay ausencia de formas- $t$ se relaciona con manifestaciones de la cortesía y con la necesidad de aprehender el carácter humano del alocutario.

Como señalé más arriba, el proceso de cambio de la vuestra merced $>$ usted ha sido explicado como una gramaticalización. Como ha sido señalado en la bibliografía, estos procesos tienen, en general, algunas o todas las características que listo a continuación: a) estratificación; b) polisemia; c) persistencia; d) ganancia de significados pragmáticos, valorativos y discursivos; e) generalización; f) desmarcación; g) obligatoriedad; h) pérdida de peso fonológico; i) debilitamiento o decoloramiento del significado léxico referencial; j) cambios de estado gramatical; k) pérdida de relacionalidad sintáctica y rigidización; 1) lexicalización.

Entiendo que si bien todas estas características se relacionan con la gramaticalización de la vuestra merced $>$ usted e iluminan los ocho siglos del proceso, me limitaré a retomar una, que me permite enriquecer la discusión de los datos con los que he trabajado.

Se trata de la persistencia (inglés "persistence"). Como han señalado Hopper (1991) esta consiste en la conservación de aspectos de la forma originaria en la forma "nueva". A mi juicio, la persistencia, las huellas que se conservan del significado primero honorífico, o reverencial de la vuestra merced $>$ usted son las que hacen que esta sea un buen recurso para los escritores cuando quieren manifestarse corteses ${ }^{21}$.

El avance del proceso de gramaticalización supone que la persistencia sea cada vez menor. Esto muestra que en tanto que los usos de las formas- $t$ son los mismos en los dos períodos, el uso de cortés de usted sujeto como estrategia de cortesía decrece marcadamente en el segundo período.

20 Agradezco a la Profesora Maria Célia Lima- Hernandes (USP, Brasil) sus comentarios sobre el análisis de los datos y sus precisiones teóricas. Los errores que persistan son de mi responsabilidad.

21 En Bertolotti (en prensa) he defendido que la iconicidad de una mayor materialidad debe considerarse también uno de los factores que hace más corteses a las formas con sujeto pronominal explícito. 
Sugiero, entonces, que el proceso de gramaticalización de la subclase usted ha avanzado entre los dos períodos estudiados ya que ha perdido comportamientos conservadores y ha adquirido comportamientos innovadores: ausencia como sujeto y marcación con lo/la como objeto.

Sin embargo este proceso no ha concluido porque siendo un pronombre de segunda no se comporta absolutamente como tal, es decir, no ha adquirido todavía los comportamientos de la clase a la cual se integra.

El concepto de categoría, que incorporan los estudios de gramaticalización es relevante para entender el fenómeno estudiado. Como señala Givón (1986) apud Company (2003: 22):

\footnotetext{
Las categorías de la lengua son en estos marcos funcionalistas espacios no discretos, sino inestables, flexibles, redefinibles y manipulables de manera creativa por los hablantes. Las categorías no tienen una conformación interna homogénea [...]. Las formas lingüísticas constituyen por lo regular un continum categorial tanto entre categorías como al interior de las mismas, con zonas focales, donde se sitúan las entradas léxicas que son mejores representantes de la categoría, el prototipo.
}

La subclase de usted no se comporta, de acuerdo con lo que nos muestra este estudio, como el centro de la categoría, representado por la subclase de tú. Por un lado, porque mantiene algunos de sus valores significativos originarios y por otro, y en mucho menor medida, porque su origen como sintagma nominal lleva a que se manifieste morfológicamente igual que la tercera persona, generando opacidades referenciales.

\section{Conclusiones}

La principal conclusión es que la subclase usted presenta una menor integración paradigmática que las otra subclases alocutivas (tú o vos). $\mathrm{Si}$ bien el cambio estudiado se inscribe en cualquiera de las dos definiciones de gramaticalización reseñadas, los datos presentados y su interpretación a través del concepto de categoría que permite la teoría de los prototipos, me lleva a proponer que el cambio vuestra merced $>$ usted no debería ser considerado concluido ya que no ha adquirido los comportamientos prototípicos de la clase: no tiene una integración paradigmática "perfecta". Usted y su paradigma no estaban "perfectamente integrados" en el siglo XIX y seguramente no lo estén tampoco actualmente ya que en muchos contextos los verbos y los clíticos resultan opacos para la interpretación. 
Desde el punto de vista metodológico, entiendo que la combinación de un análisis cualitativo con un análisis cuantitativo puede dar resultados fructíferos.

Resta por estudiar con mayor profundidad la razón por la cual la persistencia cortés se fue perdiendo a lo largo del siglo XIX. Asimismo resta por explorar si la cortesía se volvió socialmente innecesaria o se comenzó a manifestar de otra manera, como sugiere alguno de los ejemplos analizados.

Para dar mayor confiabilidad a estas conclusiones sería necesario saber si procesos similares se dieron en otras variedades del español o en lenguas cercanamente emparentadas y cómo otros procesos de gramaticalización presentan similares debilidades paradigmáticas.

\section{Referencias bibliográficas}

BERTOLOTTI, Virginia. (en prensa) "Semántica y pragmática de los usos de usted, tú y vos como sujeto en el siglo XIX en Uruguay”, en Lopes, Célia; Letícia Rebollo (org.) As formas de tratamento em português e em espanbol: variação, mudança e funçoes conversacionais. Rio de Janeiro: UFRJ.

-—. 2009. "El tratamiento pronominal singular: el objeto directo en el español del Uruguay en siglo XIX", ponencia presentada en el I Congresso Internacional de Lingüística Histórica organizado por la Universidad Federal de Bahía, 26 al 29 de julio de 2009, Salvador, Bahia en Brasil.

BROWN, Roger; GILMAN, Albert. 1960. "The Pronouns of Power and Solidarity", en Thomas. A. Sebeok (eds.) Style in Language: Cambridge, Mass: MIT Press, 253-276.

BROWN, Penelope; LEVINSON, Stephen. 1987. Some Universals in Language Usage. Cambridge: Cambridge University Press.

CANO AGUILAR, Rafael. 1997. El español a través de los tiempos. Madrid: Arco/Libros.

COMPANY COMPANY, Concepción. 2003. "La gramaticalización en la historia del español”, en Medievalia 35 Número monográfico "Gramaticalización y cambio sintáctico en la historia del español”. 3-61.

COSERIU, Eugenio. 1981. "Creatividad y técnica lingüística. Los tres niveles del lenguaje”, en COSERIU, Eugenio. Lecciones de lingüistica general. Madrid: Gredos. 269-286. DE JONGE, Bob; NIEUWENHUIJSEN, Dorien. 2009. "Formación del paradigma pronominal de las formas de tratamiento", en Concepción COMPANY COMPANY (dir.) Sintaxis histórica de la lengua española. Segunda parte: La frase nominal. Volumen 2. México: Fondo de Cultura Económica y Universidad Nacional Autónoma de México. 15751671.

FERNÁNDEZ SORIANO, Olga. 1999. "El pronombre personal. Formas y distribuciones. Pronombres átonos y tónicos”, en BOSQUE, Ignacio; DEMONTE, Violeta. (dirs.) Gramática Descriptiva de la lengua española. Entre la oración y el discurso. 1. Colección Nebrija y Bello. Real Academia Española. Madrid: Espasa. 1209-1273. 
FONTANELLA de WEINBERG, María Beatriz. (1999). "Sistemas pronominales de tratamiento usados en el mundo hispánico”, en BOSQUE, Ignacio; DEMONTE, Violeta. (dirs.) Gramática Descriptiva de la lengua española. Entre la oración y el discurso. 1. Colección Nebrija y Bello. Real Academia Española. Madrid: Espasa. 1401-1425.

GARCÍA, Érica. 1999. “Asimetría distribucional como reflejo de congruencia (con)textual” en Neuphilologische Mitelungen, 100. 455-470.

- - 2004. "Con el Rey o con el conde?: ser vs. estar en las Crónicas de Pero López de Ayala", en Neuphilologische Mitteilungen, 104. 453-482.

GIVÓN, Talmy. 1986. "Prototypes: Between Plato and Wittgenstein", en CRAIG, C. (ed.) Noun classes and categorization. Amsterdam/Philadelphia: John Benjamins. 77-103.

HOPPER, Paul. 1991. “On some principles of grammaticization”, en Traugott, Elizabeth; HEINE, Bernd. (eds.) Approches to grammaticalization. Amsterdam/Philadelphia: John Benjamins. 17- 36.

HOPPER, Paul; TRAUGOTT, Elizabeth. 1993. "Introduction”, en Grammaticalization. Cambridge:Cambridge University Press. 1-31.

HEINE, Bernd; CLAUDI, Ulrike; HÜNNEMEYER, Friedericke. 1991. Grammaticalization. A conceptual Framework. Chicago: The University of Chicago Press.

KABATEK, Johanes (ed.) 2008. Sintaxis histórica del español y cambio lingüístico: Nuevas perspectivas desde las Tradiciones Discursivas. Frankfurt/Madrid: Vervuert/Iberoamericana.

KANY, Charles. 1945. American-Spanish syntax. Chicago: University of Chicago Press.

$\mathrm{KOCH}$, Peter. 2008. "Tradiciones discursivas y cambio lingüístico: el ejemplo del tratamiento vuestra merced en español", en KABATEK, Johanes (ed.), Sintaxis histórica del español y cambio lingüistico: Nuevas perspectivas desde las Tradiciones Discursivas. Frankfurt/Madrid: Vervuert/Iberoamericana. 53-88.

LABOV, William. 1972. "On the use of the present to explain the past”, en HEILMANN, Luigi (ed.) Proceedings of the $11^{\text {th }}$ International Congress of Linguistics Bologna, Florence, Aug.28-Sept 2 1972. Bologna: Il Mulino. 226-261.

LACA, Brenda. 2006. "El objeto directo. La marcación preposicional”, en COMPANY COMPANY, Concepción. (dir.) Sintaxis histórica de la lengua española. Primera parte: La frase verbal. Volumen 1. México: Fondo de Cultura Económica, Universidad Nacional Autónoma de México. 423-478.

LAPESA, Rafael. 1959/1942. Historia de la lengua española. Madrid: Escelicer: (5ªd.)

- - 1970. "Personas gramaticales y tratamientos en español" en Revista de la Universidad de Madrid. XIX No 74. 141-167.

OESTERREICHER, Wulf. 1996. "Lo hablado en lo escrito. Reflexiones metodológicas y aproximación a una tipología”, en KOTSCHI, Thomas; OESTERREICHER, Wulf; ZIMMERMANN, Klaus. (eds.) El español hablado y la cultura oral en España e Hispanoamérica. Frankfurt/Madrid: Vervuert/Iberoamericana. 317-340.

PENNY, Ralph. 1993. Gramática bistórica del español. Barcelona: Ariel Lingüística.

SEARLE, John. 1979. Expression and meaning: Studies in the theory of speech acts. Cambridge: Cambridge University. 